\title{
Influence of symmetry breaking on the fluctuation properties of spectra
}

\author{
A Abd El-Hady, A Y Abul-Magd and M H Simbel \\ Faculty of Science, Zagazig University, Zagazig, Egypt
}

\begin{abstract}
We study the effect of gradual symmetry breaking in a non-integrable system on the level fluctuation statistics. We consider the case when the symmetry is represented by a quantum number that takes one of two possible values, so that the unperturbed system has a spectrum composed of two independent sequences. When symmetry-breaking perturbation is represented by a random matrix with an adjustable strength, the shape of the spectrum monotonously evolves towards the Wigner distribution as the strength parameter increases. This contradicts the observed behaviour of the acoustic resonance spectra in quartz blocks during the breaking of a point-group symmetry that has two eigenvalues, where the system changes in the beginning towards the Poisson statistics then turns back to the GOE statistics. This behaviour is explained by assuming that the symmetry breaking perturbation removes the degeneracy of a limited number of levels, thus creating a third chaotic sequence. As symmetry breaking increases, the new sequence grows at the expense of the initial pair until it overwhelms the whole spectrum when the symmetry completely disappears. The calculated spacing distribution and spectral rigidity are able to describe the evolution of the observed acoustic resonance spectra.
\end{abstract}

PACS: 05.45.+b, 11.30.Er, 24.60.Lz, 62.30+d 


\section{Introduction}

The statistical theory of spectra [1] provides an appropriate method for examining the symmetry properties of quantum systems. Level statistics such as the nearest-neighbour spacing (NNS) distribution and the spectral rigidity $\Delta_{3}$ are defined for a pure sequence of levels that have same quantum numbers. Usually, it is taken as granted that in a classically integrable system, which has as many integrals of motion (or quantum numbers) as the number of degrees of freedom, the levels are uncorrelated and so have a Poissonian NNS distribution. The pure level sequence in a time-reversal-invariant quantum system whose classical counterpart is chaotic, is successfully represented by a Gaussian orthogonal ensemble (GOE) of random matrices. A deviation of level statistics from the predictions of the GOE may be taken as an evidence that the sequence is not pure because of the existence of a hidden symmetry. The statistical theory of spectra has also been extensively used in the investigation of symmetry breaking because the destruction of a quantum number has a dramatic effect on the spectral fluctuations. Impressive are the studies of isospin mixing in light nuclei [2. Shriner et al. [3] measured a " complete" spectrum of low-lying states of ${ }^{26} \mathrm{Al}$ involving states with isospins $T=0$ and 1 throughout the energy range covered by the data. These data offered a testing ground for studying the influence of isospin-symmetry breaking on the fluctuation properties of energy spectra (see, e.g., 4, 5, 6, 7]). However, the limited data available in ${ }^{26} \mathrm{Al}$ (142 levels) precluded a definite conclusion. In a recent experiment with monocrystalline quartz blocks, Ellegaard et al. [8] measured about 1400 well-resolved acoustic resonances. The properties of quartz allowed these authors to measure the gradual break-up of a point-group symmetry, which is statistically fully equivalent to the breaking of a quantum number like isospin. The spectra are obtained by externally tuning the symmetry breaking [8], allowing a statistically significant investigation of the whole transition.

In the present paper we consider the effect of gradual symmetry breaking on the fluctuation properties of energy levels. When the symmetry is full the spectrum is divided into independent sequences, each corresponding to one of the eigenvalues of the symmetry operator. We consider two possible scenarios for breaking the symmetry. The first is provided by the Guhr-Weidenmüller model [5], which assumes that the perturbation acts equally on all states and that the matrix elements between states of different symmetry eigenvalues are random numbers of equal variances. In the second scenario, the perturbation acts differently on the unperturbed states and the symmetry breaking interaction mixes a limited number of the degenerate eigenstates that belong to different symmetry representations, and this number grows as we increase symmetry breaking. We are thus creating a new sequence of levels corresponding to states with no symmetry at the expense of the initial sequences. Consequently, as the fractional density of the no-symmetry sequence increases, the $\Delta_{3}$ statistics of the total spectrum first increases, moving in the direction of the prediction of the Poisson statistics until the densities of all the sequences become equal, and then decreases to reach asymptotically the value given by the GOE. Section 3 
shows that the behaviour described by the second scenario is indeed present in the data of Ellegaard et al. [8]. The summary and conclusions of this work are given in Sect. 4.

\section{Symmetry breaking in a chaotic system}

We consider a chaotic system that conserves a given symmetry. In such a system, the Hamiltonian assumes a block-diagonal form [1]:

$$
H_{S}=\operatorname{diag}\left(H_{1}, H_{2}, \cdots, H_{n}\right),
$$

where each sub-block corresponds to states of certain quantum numbers. Thus, when the symmetry is conserved, the levels are divided into a number of "pure" sequences, each described by a pure GOE. However, the statistical properties of the total spectrum that combines these sequences are no longer of the GOE type. In the limit of infinite number of sequences combined, the spectrum obeys the Poisson statistics. For a finite number of sequences, the fluctuation properties are intermediate between the Poisson and GOE statistics. In the following, we shall restrict our consideration to the case when the symmetry under investigation is represented by an operator that has two possible eigenvalues, such as parity, or isospin 0 and 1 as considered in Refs [3, 4, , 5, 6, 77.

The NNS distribution of a spectrum resulting from a random superposition of $n$ independent sequences is calculated, e.g., by Berry and Robnik [9] and in Mehta's book [10]. If the level density of the $i$ th sequence is $\rho_{i}$, and if the NNS distribution of levels of this sequence is $P_{i}\left(x_{i}\right)$, where $x_{i}=f_{i} s, f_{i}=\rho_{i} / \Sigma \rho_{i}$, and $s$ is the NNS normalised to a unit mean, then the NNS distribution of the mixed sequence is given by

$$
P(s)=E(s)\left\{\sum_{i} f_{i}^{2} \frac{P_{i}\left(f_{i} s\right)}{E_{i}\left(f_{i} s\right)}+\left[\sum_{i} f_{i} \frac{1-W_{i}\left(f_{i} s\right)}{E_{i}\left(f_{i} s\right)}\right]^{2}-\sum_{i}\left[f_{i} \frac{1-W_{i}\left(f_{i} s\right)}{E_{i}\left(f_{i} s\right)}\right]^{2}\right\},
$$

where $E(s)=\prod_{i=1}^{n} E_{i}\left(f_{i} s\right), E_{i}\left(x_{i}\right)=\int_{x_{i}}^{\infty}\left[1-W_{i}(x)\right] d x$, and $W_{i}\left(x_{i}\right)=\int_{0}^{x_{i}} P_{i}(x) d x$. In particular, if all the $n$ individual sequences have the same level densities, so that $f_{i}=1 / n$, and if the NNS distribution in each is a Wigner distribution

$$
P_{i}\left(x_{i}\right)=\frac{\pi}{2} x_{i} e^{-\frac{\pi}{4} x_{i}^{2}}
$$

which is a good approximation for the NNS distribution of a GOE, then (2) becomes 11]

$$
P_{n}(s)=\frac{1}{n}\left[\operatorname{erfc}\left(\frac{s \sqrt{\pi}}{2 n}\right)\right]^{n} Q_{n}(s)\left[\frac{\pi}{2 n} s+(n-1) Q_{n}(s)\right]
$$

where $Q_{n}(s)=\exp \left(-\pi^{2} s^{2} / 4 n^{2}\right) / \operatorname{erfc}(s \sqrt{\pi} / 2 n)$ and $\operatorname{erfc}(x)$ is the complementary error function. This distribution has a shape intermediate between those 
of the Wigner and the Poisson distributions. The Brody distribution [12],

$$
P_{\mathrm{B}}(s)=c_{\beta} s^{\beta} \exp \left(-\frac{c_{\beta}}{\beta+1} s^{\beta+1}\right) \quad \text { with } \mathrm{c}_{\beta}=\frac{\Gamma^{\beta+1}(1 /(\beta+1))}{\beta+1},
$$

accurately reproduces the spectra of many two-dimensional systems with mixed regular-chaotic dynamics. As we vary the parameter $\beta$ from 0 to 1 the statistics changes from Poissonian to GOE.

The NNS distribution contains information about the spectrum in a short range, not exceeding a maximum of three mean level spacings. Long range information is provided by various higher-order correlation functions [1], 10]. The most popular among these is the spectral rigidity $\Delta_{3}$ of Dyson and Mehta [13]. Semiclassical arguments [14] show that, for a generic integrable system whose spectrum satisfies Poisson statistics

$$
\Delta_{3, \text { Poisson }}(L)=\frac{L}{15} \text { for } \mathrm{L} \ll \mathrm{L}_{\max },
$$

where $L_{\max }=2 \pi \hbar / D T_{\min }, D$ is the mean level spacing and $T_{\min }$ is the period of the shortest classical orbit. For time-reversal-invariant chaotic system the semiclassical theory gives the following asymptotic expression

$$
\Delta_{3, \mathrm{GOE}}(L)=\frac{1}{\pi^{2}} \ln L-0.007 \text { for } 1 \ll \mathrm{L} \ll \mathrm{L}_{\max } .
$$

In all cases $\Delta_{3}$ saturates to a non-universal value at $L \sim L_{\text {max }}$. The random matrix theory obtains an analytical expression for $\Delta_{3, \mathrm{GOE}}(L)$ that involves double integration [1, 10]. We find it more suitable to parametrise this expression for a GOE, guided by Eq. (耳), in the form

$$
\Delta_{3, \mathrm{GOE}}(L)=\frac{1}{\pi^{2}}\left(1-e^{-a L}\right)[\ln (L+b)+c],
$$

with $a=8.241, b=0.944$ and $c=-0.06724$ being the best fit parameters for values of $L$ in the range of $0 \leq L \leq 50$. Seligman and Verbaarschot 15] suggested the following expression for the spectral rigidity of a spectrum resulting from a random superposition of $n$ independent sequences with fractional level densities $f_{i}$

$$
\Delta_{3}(L)=\sum_{i=1}^{n}\left[\Delta_{3, i}\left(f_{i} L\right)\right]
$$

where $\Delta_{3, i}(L)$ is the spectral rigidity for the $i$ th sequence. This proposal is not surprising because $\Delta_{3}(L)$ is essentially a variance.

We now consider symmetry breaking in the chaotic system using the GuhrWeidenmüller model [5]. The model suggests that the effect of the symmetrybreaking perturbation on all the unperturbed states is equal on the average. This suggests defining the Hamiltonian in the form:

$$
H=\left[\begin{array}{cc}
H_{0} & 0 \\
0 & H_{0}
\end{array}\right]+\alpha\left[\begin{array}{cc}
0 & W \\
W^{T} & 0
\end{array}\right]
$$


where $H_{0}$ represents a GOE and $W$ is a random matrix whose elements have Gaussian distributions with zero means and variances equal to those of the nondiagonal elements of $H_{0}$. When $\alpha=0$, the symmetry is conserved and the Hamiltonian has the block diagonal form of Eq. (1) with $n=2$. The spectrum consists of an independent superposition of two level sequences having equal density. The NNS distribution $P(s)$ is given by Eq. (任) and, in particular, $P(0)=1 / 2$. The spectral rigidity is given by (9) with $f_{1}=f_{2}=1 / 2$. When we allow $\alpha$ to increase, the system gradually evolves toward a single GOE. To show how this transition proceeds, we considered an ensemble of ten $200 \times 200$ Hamiltonian matrices of the form (10) for a fixed value of the perturbation strength $\alpha$. We numerically diagonalised these matrices. We obtained the NNS distribution and spectral rigidity for each of the resulting spectra, and measured their deviation from the GOE statistics by comparing with the Brody distribution, Eq. (5), for the NNS distribution and with the Seligman and Verbaarschot expression, Eq. (9), for the $\Delta_{3}$ statistics where $\Delta_{3,1}$ is given by the Poisson distribution (6) and $\Delta_{3,2}$ by the GOE (8), so that

$$
\Delta_{3, \mathrm{SV}}(L)=q_{\mathrm{SV}} \frac{L}{15}+\Delta_{3, \mathrm{GOE}}\left[\left(1-q_{\mathrm{SV}}\right) L\right],
$$

We then repeated the procedure for other value of $\alpha$. The best fit of the tuning parameters $\beta$ and $q_{S V}$ obtained for $P(s)$ and $\Delta_{3}(L)$ corresponding to different values of the perturbation strength $\alpha$ are shown in Fig. 1. The error bars measure the changes of the parameters that increase the minimum $\chi^{2}$ values by a factor of 2 . The monotonous nature of the curves clearly shows that the transition from symmetry to no-symmetry in the Guhr-Weidenmüller model is gradual and one-directional.

The opposite extreme of the Guhr-Weidenmüller model is the case when the eigenfunctions will become either very weakly or very strongly perturbed. For those states in which the perturbation is weak enough, the wave function can in principle be characterised by one of the two symmetry eigenvalues. These will be the states for which the splitting of two degenerate levels belonging to the two symmetry representations can be neglected. The corresponding energy levels will again form two independent sequences with fractional level densities $f_{1}=f_{2}=f / 2$, while the levels of the strongly perturbed eigenstates will form a new sequence with density $f_{3}=1-f$ which will grow at the expense of the other two as the symmetry-breaking perturbation increases. The NNS distribution will then be given, using (2) and (3), by

$$
\begin{aligned}
P(s)= & \frac{\pi}{8} f^{3} s e^{-\pi f^{2} s^{2} / 16} \operatorname{erfc}\left(\frac{\sqrt{\pi}}{4} f s\right) \operatorname{erfc}\left[\frac{\sqrt{\pi}}{2}(1-f) s\right] \\
& +\frac{1}{2} f^{2} e^{-\pi f^{2} s^{2} / 8} \operatorname{erfc}\left[\frac{\sqrt{\pi}}{2}(1-f) s\right] \\
& +\frac{\pi}{2}(1-f)^{3} s e^{-\pi(1-f)^{2} s^{2} / 4}\left[\operatorname{erfc}\left(\frac{\sqrt{\pi}}{4} f s\right)\right]^{2}
\end{aligned}
$$




$$
+2 f(1-f) e^{-\pi\left(5 f^{2}-8 f+4\right) s^{2} / 16} \operatorname{erfc}\left(\frac{\sqrt{\pi}}{4} f s\right) .
$$

for spacings greater than a certain lower limit. If we ignore the latter condition, the extrapolation of $P(s)$ into the origin yields

$$
P(0)=f\left(2-\frac{3}{2} f\right)
$$

We note that $P(0)$ increases with decreasing $f$ from a value of $1 / 2$ at $f=1$, reaches a maximum value of $2 / 3$ at $f=2 / 3$, and then decreases monotonously until it vanishes at $f=0$. Equations (12) and (13) show that the evolution of the shape of the NNS distributions with increasing symmetry breaking, and decreasing $f$, is not a straightforward transition from the 2-GOE behaviour to that of a GOE. Indeed, by decreasing the value of $f$ we are removing some of the levels from the initial two symmetry-invariant sequences to form the third sequence that represents the states without symmetry. The distribution will look more regular when we decrease $f$ until the level densities of the three sequences become equal. If we further increase the symmetry breaking, the initial sequences become thinner until the whole spectrum forms a single GOE sequence.

We can follow this transition better by calculating the spectral rigidity which is, for this system, given by

$$
\Delta_{3}(L)=2 \Delta_{3, \mathrm{GOE}}\left(\frac{f L}{2}\right)+\Delta_{3, \mathrm{GOE}}[(1-f) L] .
$$

In Fig. 2, the spectral rigidities corresponding to $f=1,0.9,0.667,0.333,0.1$ and 0 are given by the curves labelled by A, B, C, D, E and F, respectively, while the curve $\mathrm{P}$ corresponds to a regular spectrum. The figure clearly shows that $\Delta_{3}(L)$ increases as symmetry breaking increases, and thus evolves towards the regular shape until $f$ reaches the value of $2 / 3$. Only then starts $\Delta_{3}(L)$ a route leading to the GOE curve.

\section{Comparison with acoustic resonance spectra}

In this section, we show that the proposed application of the Berry-Robnik theory to symmetry breaking leads to a satisfactory description of the acoustic resonance spectra of monocrystalline quartz blocks, measured by Ellegaard et al. [8]. Crystalline quartz exhibits $D_{3}$ point-group symmetry about the crystal's $Z$ (optical) axis, and three two-fold rotation symmetries about the three $X$ (piezoelectric) axes; the latter three axes lie in a plane orthogonal to the $Z$ axis and sustain angles of 120 degrees with respect to each other. Ellegaard et al. used rectangular blocks of dimensions $14 \times 25 \times 40 \mathrm{~mm}^{3}$, cut in such a way that all symmetries are fully broken except a two-fold "flip" symmetry about one of the three $X$ axes. A gradual breaking of this symmetry is then achieved by removing successively bigger octants of a sphere from one corner, thereby 
creating a three-dimensional Sinai billiard. The acoustic spectrum is measured for 8 radii of the removed octant, providing data of high statistical significance for the study of the gradual breaking of a point-group symmetry.

Figure 3 shows the spectral rigidity for the cases when the symmetry is present (open circles), and when it is violated by removing a tiny octant of radius $r=0.5 \mathrm{~mm}$ (closed circles). The straight line labelled $\mathrm{P}$ is for an integrable system. We note that data for the case of tiny symmetry violation are systematically higher than the ones for the case of conserved symmetry. This agrees with the prediction of the second scenario proposed above, in which the system that undergoes a gradual symmetry breaking looks as if it has become more regular in the early stages of the transition.

Ellegaard et al. 8] concluded from the rise of $\Delta_{3}$ over the theoretical prediction for a superposition of two GOE sequences that the quartz block with conserved flip symmetry has much in common with a pseudo-integrable system. They supported this conclusion by means of other measurement. Pseudointegrable (PI) systems are non-integrable systems, yet non-chaotic. Twodimensional PI systems, exemplified by particles moving in a planar polygonal enclosure with rational angles [16], share with the integrable system that the motion of trajectories in the phase space is restricted to two-dimensional compact surfaces. But these invariant surfaces are multi-connected and not tori as in the case of integrable systems. Numerical studies of the $\pi / 3$ rhombus billiard [17, 18, 19] show that the spectral fluctuation properties are intermediate between those of a Poisson ensemble and a GOE. Bogomolny et al. [19] studied the level statistics of PI systems using a modified version of Dyson's stochastic Coulomb gas model [10], in which the interaction between particles is restricted only to the nearest neighbours. The NNS distribution obtained in 19] is given by

$$
P_{\mathrm{PI}}(s)=4 s e^{-2 s} .
$$

This distribution is in agreement with the numerical calculation for the rational billiards 18. Unfortunately, the formula obtained by these authors for the two-point correlation function

$$
R_{2}(s)=1-4 e^{-4 s}
$$

leads to an expression for the $\Delta_{3}$ statistics that asymptotically increases as $L / 30$, which is not consistent with the results of the numerical experiments. Following several authors, e.g. [17], we shall represent the $\Delta_{3}$ statistics by Seligman-Verbaarschot [15] formula (9), obtained for a superposition of Poisson and GOE level sequences,

$$
\Delta_{3, \mathrm{PI}}(L)=q \frac{L}{15}+\Delta_{3, \mathrm{GOE}}[(1-q) L]
$$

where $q$ is a fitting parameter. Biswas and Jain [17 found $q$ to be equal to 0.2 for the $\pi / 3$ rhombus billiard.

We now apply the second scenario, which has been proposed in the previous section, to the symmetry breaking in a PI system having a symmetry represented by a quantum number that takes one of two possible values. When 
the symmetry is conserved, the spectrum is an independent superposition of two sequences corresponding to the two representations of the symmetry. The NNS distribution is accordingly represented by (2) and the $\Delta_{3}$ statistics by (9) with $n=2, P_{i}\left(x_{i}\right)$ given by (15) and $\Delta_{3, i}(L)$ by (17). We now again assume that as the symmetry violation starts, the eigenfunctions of the system will be divided into two classes. The first constitutes a fraction $f$ of the eigenfunctions in which the perturbation due to symmetry breaking can be ignored. The levels of these eigenstates are divided into two independent sequences exactly as before switching the symmetry breaking on. The second is the class of the strongly perturbed states that have completely lost the symmetry. This latter class will here be modelled by a GOE. Therefore, the total spectrum will be composed of 3 independent sequences, two with PI statistics and each having a fractional density of $f / 2$, and one with GOE statistics and fractional density $1-f$. During the symmetry-breaking transition, the NNS distribution is given by

$$
\begin{aligned}
P(s)= & \frac{1}{2} f^{2}\left(2 f^{2} s^{2}+4 f s+1\right) e^{-2 f s} \operatorname{erfc}\left[\frac{\sqrt{\pi}}{2}(1-\mathrm{f}) \mathrm{s}\right] \\
& +\frac{1}{8}(1-f)(2+f s) \times\left[\pi f s^{2}(1-f)^{2}+2 s\left\{(\pi+4) f^{2}-2 \pi f+\pi\right\}+8 f\right] \\
& \times e^{-\frac{\pi}{4}(1-f)^{2} s^{2}-2 f s},
\end{aligned}
$$

which is obtained by substituting Eqs. (3) and (15) into Eq. (2). The $\Delta_{3}$ is given by

$$
\Delta_{3}(L)=2 \Delta_{3, \mathrm{PI}}\left(\frac{f L}{2}\right)+\Delta_{3, \mathrm{GOE}}[(1-f) L] .
$$

We now compare our model with the acoustic resonance spectra measured by Ellegaard et al. $[8]$. We start by estimating the parameter $q$ in the expression (17) for the $\Delta_{3}$ statistics of the PI system. We do this by fitting the same statistics for the spectrum of the complete quartz cube to Eq. (19) with $f=1$ and obtain $q=0.082$. We then compare Eq. (19) with the spectral rigidities of the other spectra considering $f$ as a free parameter. The best-fit values of $f$ for spectra corresponding to different radii of the removed octants are given in Table 1. We use these values to calculate the NNS distributions. The results of the calculation are compared with the experimental data in Fig. 4. We see that the proposed model presents a satisfactory description of the whole transition by varying a single parameter. The only disagreement is between the calculated and measured values of $P(s)$ at small values of $s$ as we have already expected. The symmetry breaking decreases the probability of finding degenerate levels sharply leading to the observed dip at small $s$ in the spacing distributions [8]. This dip is followed by an overshoot to restore normalisation. The width of this dip, which is about $1 / 10$ of the mean level spacing, provides an estimate for the minimum level-splitting below which two levels can be regarded as approximately degenerate. 


\section{Summary and conclusions}

Conventional models for symmetry breaking in chaotic systems assume that the non-diagonal matrix elements of the symmetry breaking perturbation are statistically equivalent. They predict a monotonous transition from a mixed statistics to the GOE as one switches on and increases the symmetry-breaking perturbation. This is not the behaviour of the acoustic resonance spectra of quartz measured by Ellegaard et al $[8]$. We propose another scenario for symmetry breaking in which the levels are approximately separated into two groups, one with and one without symmetry. This happens when the expansion of any of the wavefunctions in terms of the eigenfunctions of the symmetry operator is either dominated by a single term or composed of statistically equivalent contributions from all its component. Considering a system in which a symmetry that has two representations is conserved, we represent the spectrum as a superposition of two independent sequences, one for each symmetry representation. Now, as we switch on the symmetry breaking perturbation, we remove a number of levels from these two level sequences into a third sequence. The latter corresponds to the strongly perturbed states in which the symmetry has virtually disappeared. Because we have increased the number of spectral partitions, the spectrum will look as if it became more regular by the small violation of the symmetry. The apparent regularity will continue until the three sequences become equally populated. The further increase of symmetry breaking will reverse the evolution of the shapes of the spectral statistics toward the GOE shapes. Namely this is the behaviour found by inspection of the acoustic resonance spectra of quartz measured by Ellegaard et al [8]. We see, for instance, from Fig. 4 that it is possible to describe the evolution of the NNS distribution and the spectral rigidity during the transition from a fully conserved symmetry to a fully violated one by varying a single parameter, measuring the fractional density of states which are practically unaffected by symmetry violation. 


\section{References}

[1] Guhr T, Müller-Groeling A and Weidenmüller H A 1998 Phys.Rep. 299 189

[2] Harney H L, Richter A and Weidenmüller H A 1986 Rev. Mod. Phys.. 58 607

[3] Shriner J F, Jr., Bilpuch E G, Endt and Mitchell G E 1990 Z.Phys. 335 393

[4] Paar V, Vorcapic D, van Hees A G M and Wolters A A 1989 Phys. Rev. Lett. 622643

[5] Guhr T and Weidenmüller H A 1990 Ann. Phys. (N.Y.) 199412

[6] Hussein M S and Pato M P 1993 Phys. Rev. C 47412

[7] Abul-Magd A Y and Simbel M H 1997 Phys. Rev. C 56825

[8] Ellegaard C, Guhr T, Lindemann K, Nygård J and Oxborrow M 1976 Phys. Rev. Lett. 774918

[9] Berry M V and Robnik M 1984 J. Phys. A: Math. Gen. 172413

[10] Mehta M L 1991 Random Matrices 2nd ed. (New York: Academic)

[11] Abul-Magd A Y and Simbel M H 1996 Phys. Rev. E 543293

[12] Brody T A 1973 Lett. Nuovo Cimento 7482

[13] Dyson F M and Mehta M L 1963 J. Math. Phys. 4701

[14] Berry M V 1985 Proc. R. Soc. London Ser. A 400229

[15] Seligman T H and Verbaarschot J J M 1985 J. Phys. A: Math. Gen. 18 2227

[16] Richens P J and Perry M V 1981 Physica D 2495

[17] Biswas D and Jain S R 1990 Phys. Rev. A 423170

[18] Grémaud B and Jain S R 1998 J. Phys. A: Math. Gen. 31 L637

[19] Bogomolny E B, Gerland U and Schmidt C 1999 Phys. Rev. E 59 R1315 
Table 1. Best fit values for the fraction $f$ of states not affected as a result of violating the point-group symmetry of a quartz rectangular block by removing spherical octants of various radii $r$. The values of $f$ are evaluated by fitting the spectral rigidities $\Delta_{3}(L)$ of Fig. 4. to Eq. (19).

\begin{tabular}{|l|l|l|l|l|l|l|l|}
\hline$r$ & 0 & $0.5 \mathrm{~mm}$ & $0.8 \mathrm{~mm}$ & $1.1 \mathrm{~mm}$ & $1.4 \mathrm{~mm}$ & $1.7 \mathrm{~mm}$ & huge defocusing \\
\hline$f$ & 1 & 0.49 & 0.37 & 0.28 & 0.23 & 0.14 & 0 \\
\hline
\end{tabular}

\section{Figure Caption}

Figure 1. The monotonous dependence of (a) the parameter $\beta$ in the Brody distribution (Eq.(5)) for the NNS distributions $P_{\mathrm{B}}(s)$ and (b) the parameter $q_{S V}$ in the Seligman and Verbaarschot expression (Eq.(11)) for the spectral rigidity $\Delta_{3}(L)$ that interpolate between the Poisson and GOE statistics, obtained by a numerical experiment on a chaotic system undergoing a gradual breaking of a symmetry according to the Guhr-Weidenmüller model [5].

Figure 2. The spectral rigidity $\Delta_{3}(L)$ for a chaotic system undergoing a gradual breaking of a symmetry according to the scenario proposed in the text. The results of the calculation when the fraction of states remaining nearly symmetry invariant $f=1,0.9,0.667,0.333,0.1$ and 0 are given by the curves labelled by A, B, C, D, E and F, respectively, while the curve P corresponds to a regular spectrum.

Figure 3. The spectral rigidity $\Delta_{3}(L)$ measured in [8] for the quartz block when the symmetry is present (open circles), and when it is violated by removing a tiny octant of radius $r=0.5 \mathrm{~mm}$ (closed circles). The straight line labelled $\mathrm{P}$ is for an integrable system.

Figure 4. The NNS distributions $P(s)$ and spectral rigidities $\Delta_{3}(L)$ for the different radii of the octant removed from a quartz block: (a) $r=0$, the flip symmetry is fully conserved, (b) $r=0.5 \mathrm{~mm}$, (c) $r=0.8 \mathrm{~mm}$, (d) $r=1.1$ $\mathrm{mm}$, (e) $r=1.4 \mathrm{~mm}$, (f) $r=1.7 \mathrm{~mm}$, (x) the block with the huge defocusing structure. The data are from Ref. 8, while the curves are the theoretical curves using Eqs. (18) and (19). Values of the parameter $f$ that measures the fractional densities of states that keep the symmetry are given in Table 1. 

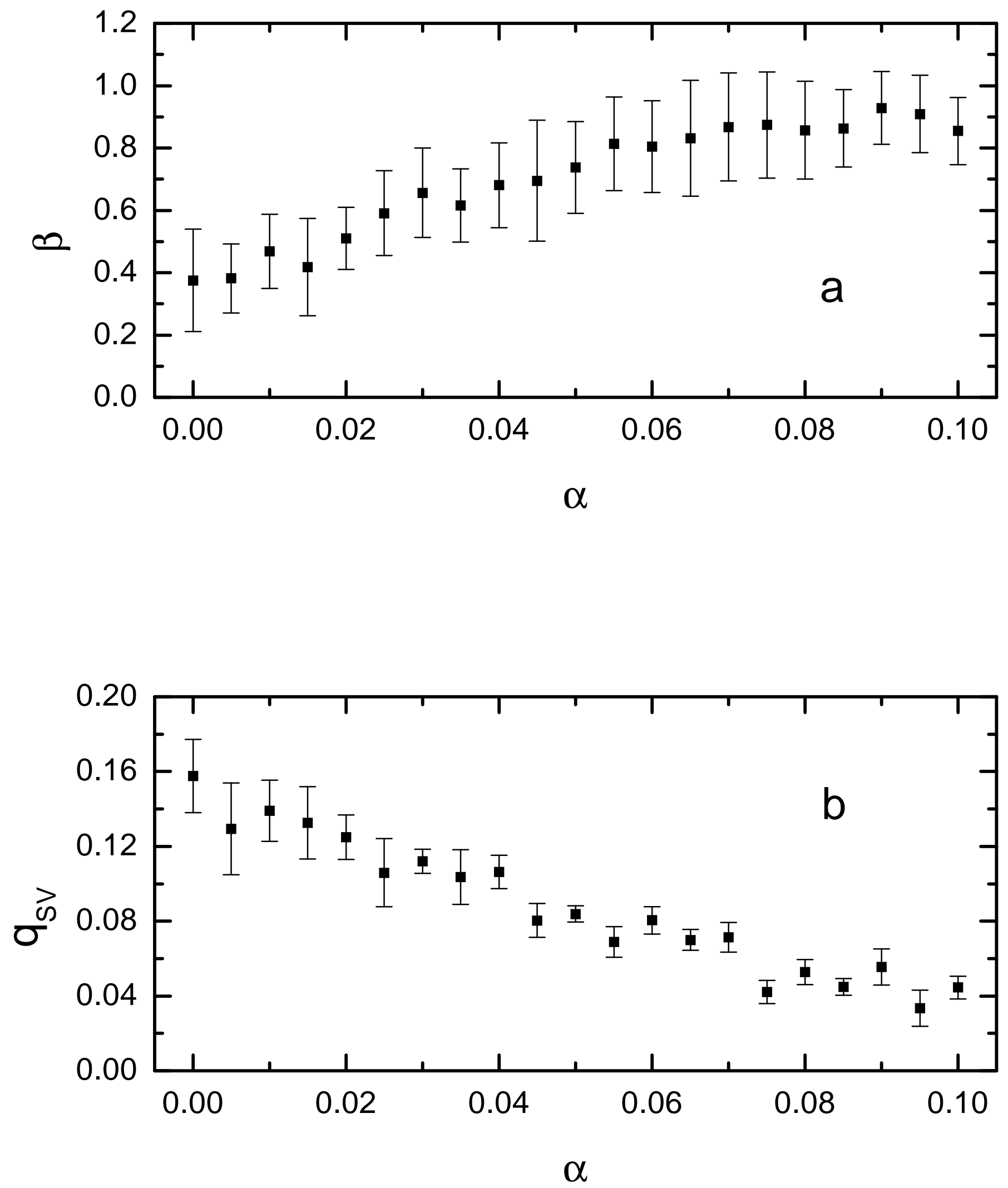

Figure 1. 


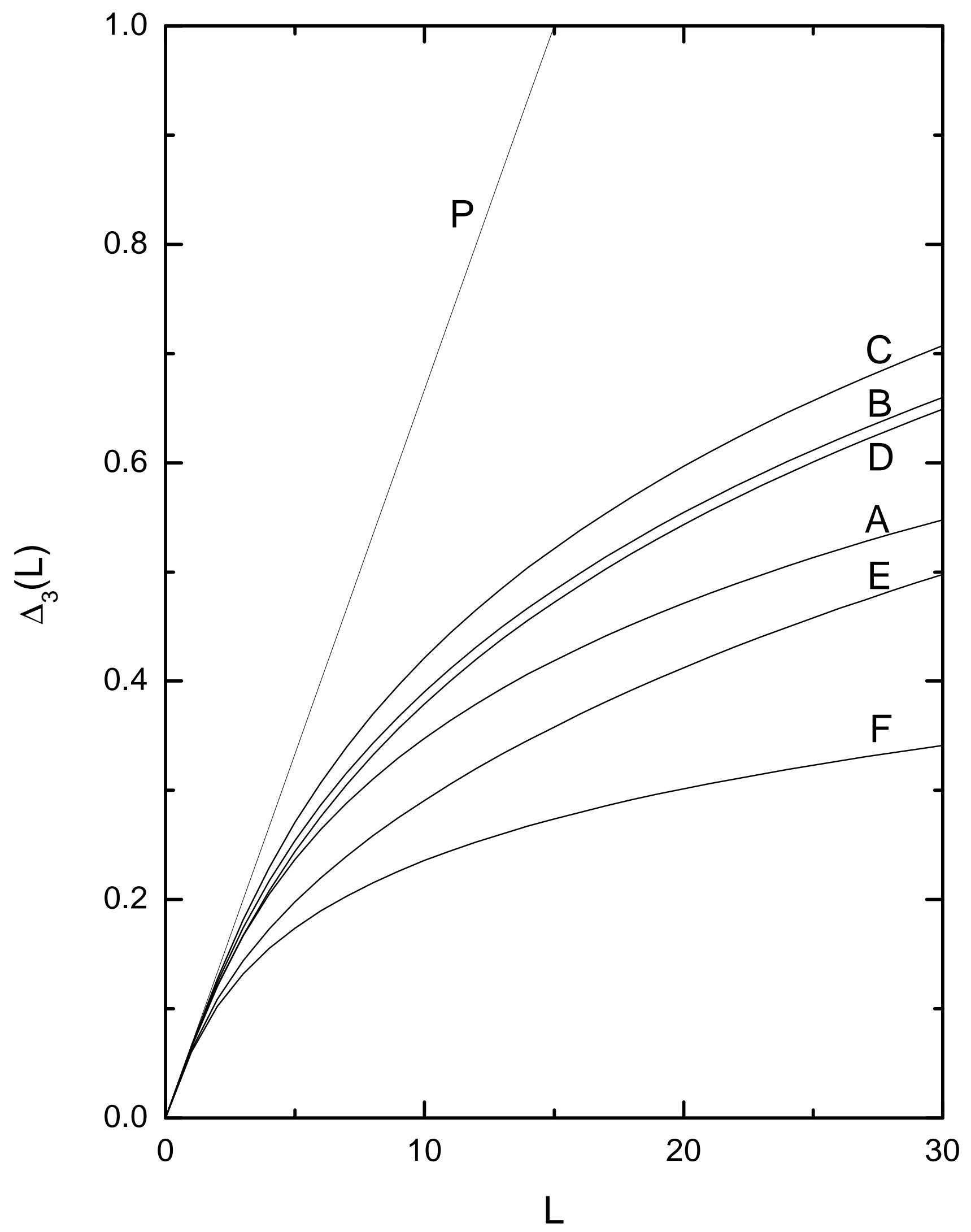

Figure 2. 


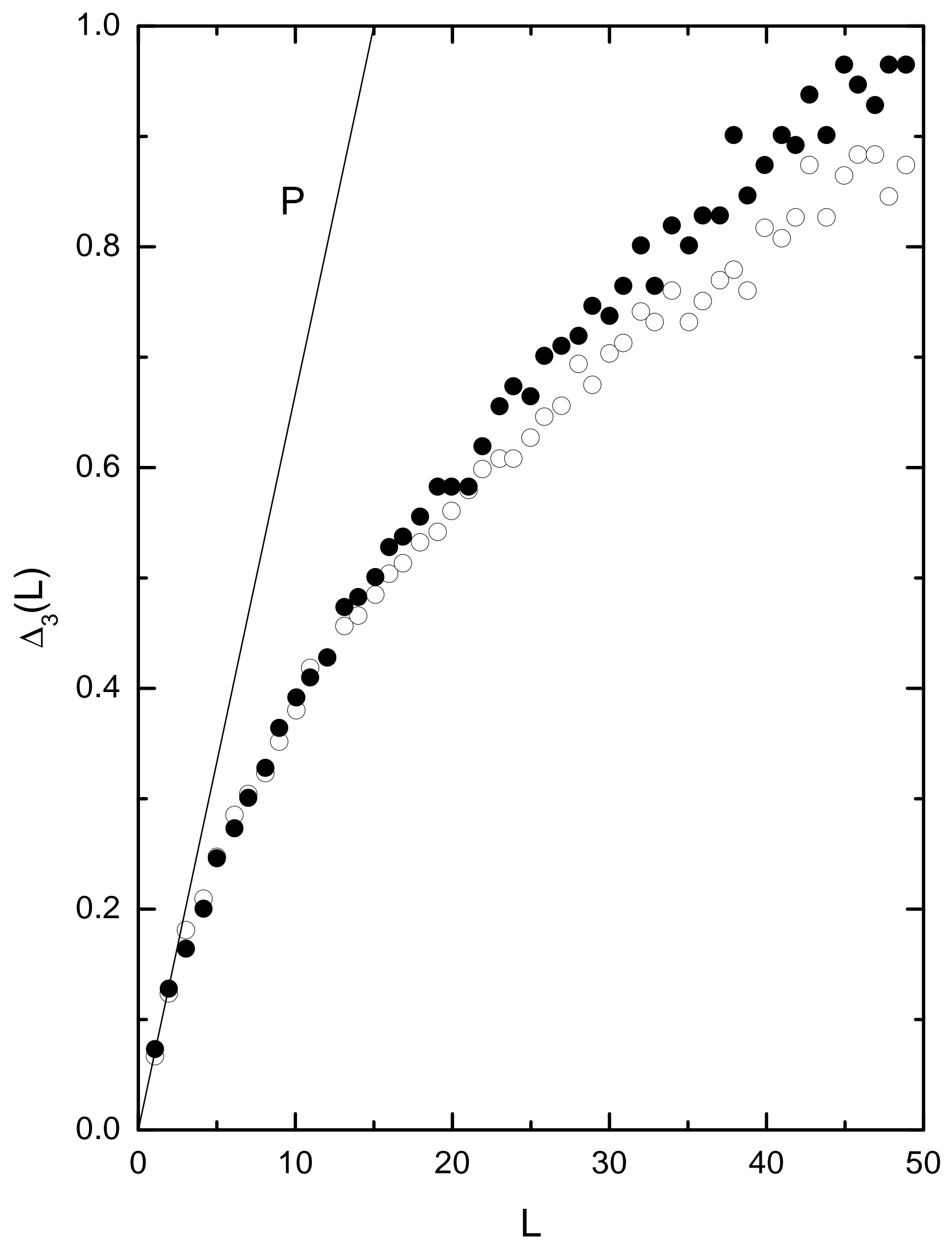

Figure 3. 


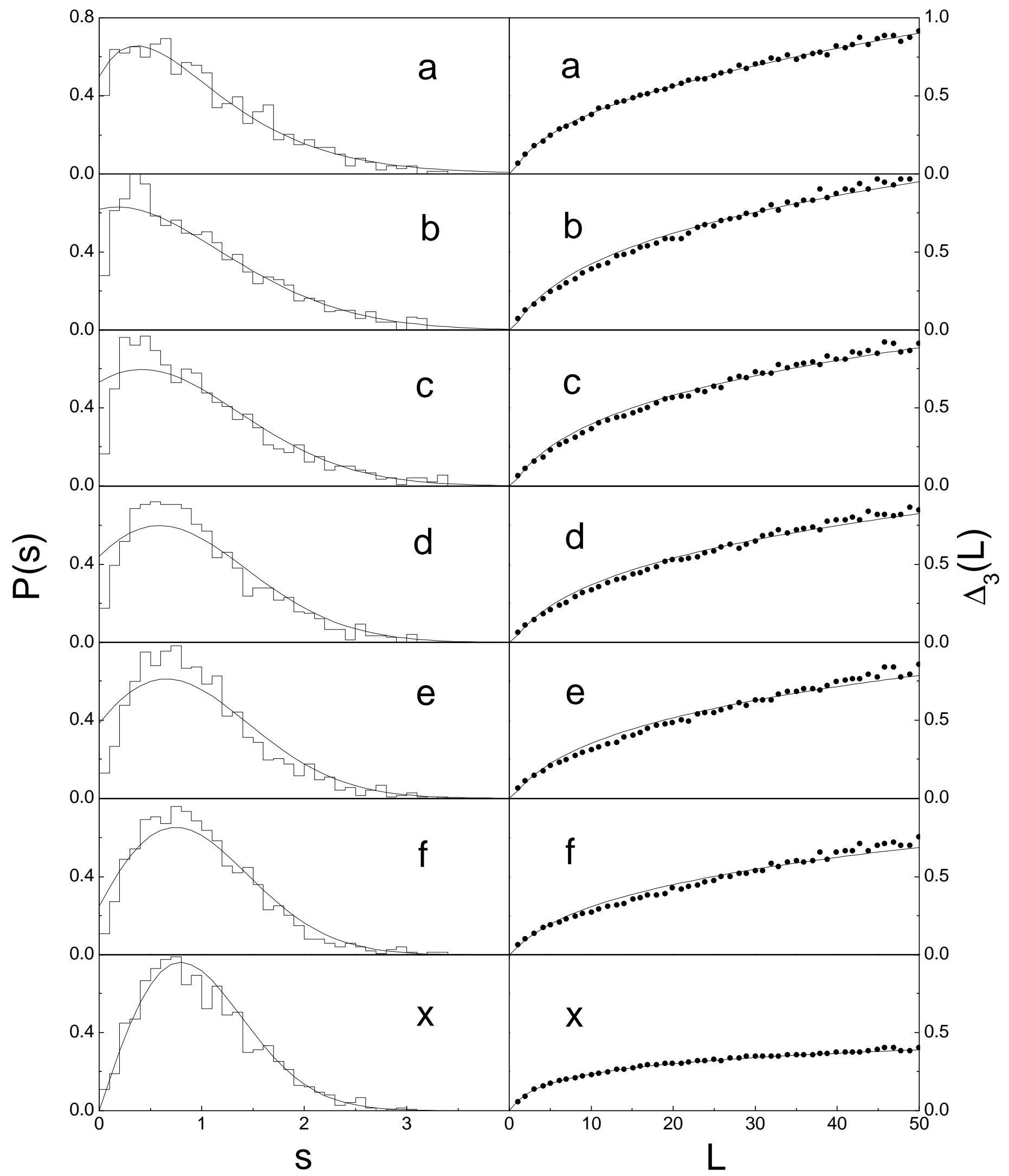

Figure 4. 